\title{
Creencias, posverdad y política ${ }^{1}$
}

\section{Beliefs, post-truth and politics}

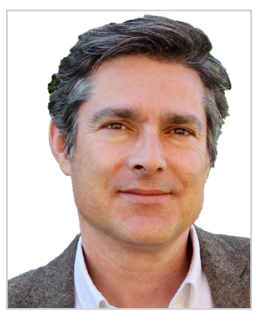

Ignacio Blanco Alfonso. Profesor titular de Periodismo en la Universidad CEU San Pablo. Se doctoró con una tesis sobre los Géneros periodísticos en la obra de José Ortega y Gasset (premio extraordinario de Doctorado, 2003). Especialista en el pensamiento y la obra del filósofo madrileño, formó parte del equipo de edición e investigación de sus Obras completas. En 2008 fundó el Máster Universitario en Periodismo Cultural. Actualmente, el profesor Blanco es Investigador Principal del PROVULDIG, proyecto financiado por la Comunidad de Madrid y el Fondo Social Europeo para crear una red de investigadores de varias universidades españolas interesados en actividades de vulnerabilidad digital.

Universidad CEU San Pablo, Madrid, España

iblanco@ceu.es

ORCID: 0000-0002-2595-464X

\section{Resumen:}

El presente texto avanza las ideas preliminares de una investigación en desarrollo sobre la influencia de las creencias personales en la formación de la opinión pública y su relación con la política en contextos dominados por la posverdad. Los acontecimientos políticos de 2016 (el Brexit y la victoria de Donald Trump) han evidenciado la vulnerabilidad de nuestras democracias frente a la comunicación política sesgada y malintencionada. La era de la posverdad ha revelado la capacidad del discurso político pasional (basado en el pathos) para arrasar con el discurso político racional (basado en el logos).

Palabras clave:

Creencias, disonancia cognitiva, posverdad, discurso político.

\section{Abstract:}

This text presents the preliminary ideas of an investigation in progress related to the influence of personal beliefs in shaping public opinion and its relationship to politics within contexts dominated by posttruth. The political events of 2016 (Brexit and the victory of Donald Trump) have demonstrated the vulnerability of our democracies with regard to biased and malicious political communications. The post-truth era has revealed the ability of passionate political discourse (based on pathos) to ravage rational political speech (based on logos).

\section{Keywords:}

Beliefs, cognitive dissonance, post-truth, political discourse.

Este trabajo se encuadrada dentro del "Programa de Actividades sobre Vulnerabilidad Digital" (PROVULDIG-CM), referencia S2015/HUM-3434, financiado por la Comunidad de Madrid y el Fondo Social Europeo. También forma parte de la investigación realizada por el autor en el Departamento de Sociología de la Universidad de Cambridge (Reino Unido), financiada por el Programa de Ayudas a la Movilidad Internacional de Profesores e Investigadores Senior, del Ministerio de Educación Cultura y Deporte, referencia PRX16/00612.

Cómo citar este artículo:

Blanco Alfonso, I. (2018). Creencias, posverdad y política. Doxa Comunicación, 27, pp. 421-428.

https://doi.org/10.31921/doxacom.n27a20 


\section{Introducción}

El 9 de noviembre de 2016, gran parte del mundo recibía con estupefacción la noticia de la victoria de Donald Trump en las elecciones presidenciales de Estados Unidos. Las crónicas y editoriales de los main stream media subrayaban el vuelco que se había producido en los sondeos de opinión que, durante meses, auguraron la victoria de la candidata del Partido Demócrata, Hilary Clinton. El día anterior a la cita electoral, el 8 de noviembre, la diferencia en las encuestas superaba los tres puntos a favor de Clinton (46,8 a 43,6) (Real Clear Politics, 2016).

Unos meses antes, el 23 de junio de 2016, Reino Unido había sacudido la opinión pública internacional con la decisión de abandonar la UE. Contra todo pronóstico, los partidarios del Brexit se impusieron con el 51,9\% de los sufragios emitidos en referéndum. Sin embargo, las encuestas habían mantenido hasta el último día la victoria de los remainers con un $48 \%$ frente a un $46 \%$ de los votos (Financial Times, 2016).

Todas las publicaciones de referencia internacional se han ocupado profusamente de ambos vuelcos electorales. En una somera revisión de las principales cabeceras estadounidenses observamos que, más allá de sus consecuencias históricas, el Brexit supuso un aldabonazo para los analistas de aquel país, toda vez que Donald Trump había sido designado candidato por el Partido Republicano. La eventualidad de que pudiera ganar las elecciones presidenciales suscitó numerosos análisis que correlacionaban ambos acontecimientos, y desde los primeros momentos dicha correlación se estableció en torno a la irrupción en el debate público de las noticias falsas (fake news) y el contexto de posverdad (post-truth) que habían inundado la campaña del Brexit y que ya estaban contaminando la campaña política de las Presidenciales que se celebrarían en noviembre de 2016.

En agosto de 2016, The New York Times denunciaba abiertamente en el artículo titulado por Williams Davies "The age of post-truth politics" las informaciones inexactas, incompletas o directamente falsas difundidas durante la campaña del Brexit, alertaba del "declive de la autoridad de los hechos" y reclamaba para los periódicos el "deber de resistencia frente a la demagogia populista" (Davies, 2016).

En la misma línea, The Economist editorializaba en septiembre de 2016 sobre el riesgo de las noticias falsas y la creación de un estado de posverdad política que podría desembocar en acontecimientos similares al Brexit. En "Art of the lie", The Economist alertaba del riesgo de este tipo de campañas políticas basadas en los sentimientos, no en los hechos, campañas que, como en el caso del Brexit, obligan a los oponentes a "luchar en terrenos que ellos no han elegido" (The Economist, 2016).

El director de The New Yorker, David Remnick, inauguró en septiembre de 2016 una serie de reportajes en profundidad titulada "Trump and the Truth". Remnick anunciaba la puesta en marcha de los departamentos de fact-cheking (verificación de datos) no solo en The New Yorker, sino también en cabeceras como The New York Times, The Washignton Post y en el sitio especializado en verificación de datos Politifact, cuyos reporteros asumían la función de corroborar la exactitud de las noticias prominentes de la campaña electoral para orientar a la opinión pública en algunos de los temas centrales, como la inmigración, el crimen, el desempleo o las teorías de la conspiración (The New Yorker, 2016).

En diciembre de 2016, habiéndose consumado la victoria de Donald Trump, la responsable de la página editorial de The Washington Post, Ruth Marcus, titulaba su comentario "Wellcome to the post-truth presidency" (The Washington Post, 
2016). Como en los casos anteriores, este periódico denunciaba el "caso omiso" del presidente electo hacia los hechos y los datos verificados, y señalaba que Trump y sus asesores "abrazan” la posverdad sin ningún tipo de reparo.

Unos meses más tarde, la experta en Rusia Masha Gessen publicaba un extenso análisis en The New York Review of Books titulado "The Putin Paradigm”, donde establecía un paralelismo entre Putin y Trump basado en la convicción compartida por ambos líderes de que "la mentira es el mensaje" (The New York Review of Books, 2016).

Esta breve muestra editorial de algunos de los medios internacionales de referencia nos sirve para señalar la disonancia entre la realidad factual (lo que ha pasado) y la realidad mediática representada en el discurso periodístico dominante (lo que se decía que iba a pasar).

Los votos emitidos a favor de Trump y del Brexit han evidenciado una voluntad popular -ligeramente mayoritaria en ambos países- disonante con el discurso de los medios de referencia, por lo que cabe preguntarse: ise trata de un error de interpretación de los indicios por parte de analistas y expertos? ¿ Hubo señales que apuntaban en la dirección de los resultados finales que, sin embargo, se obviaron?

\section{Posverdad y opinión pública}

No es aventurado pensar que el concepto de posverdad ha surgido en nuestra agenda por la necesidad de disponer de un recurso lingüístico performativo que nos permita describir el signo que está adoptando la democracia deliberativa del siglo XXI. Su uso se multiplicó un 2000\% durante 2015, de ahí que el English Oxford Dictionary la seleccionara palabra del año: "Relating to or denoting circumstances in which objective facts are less influential in shaping public opinion than appeals to emotion and personal belief” (Oxford Dictionary, 2016).

La correlación entre los conceptos de opinión pública y emociones o convicciones personales recuerda, inevitablemente, a la literatura científica del primer tercio del siglo XX, cuando entra en crisis la llamada “sociedad de masas" (Ortega y Gasset, 1930) y se toma conciencia del poder de la comunicación social como una especie de "gobierno en la sombra" (Bernays, 1928), en el que unos "ingenieros en la sombra" (Lasswell, 1935) serían capaces de dirigir las decisiones individuales y de "manufacturar el consentimiento" (Lippmann, 1922). La idea -que en muchos casos supuso la aplicación empírica de los fundamentos teóricos de Freud sobre el subconsciente- consistía en que, en un clima social debidamente moldeado por la acción de la propaganda, los razonamientos construidos sobre los hechos verificados (logos) serían arrasados por mensajes simbólicos que apelaran a emociones primarias del ser humano (pathos), mermando su análisis racional y provocando respuestas emocionales.

En el subsuelo de estas ideas había germinado la desconfianza hacia la capacidad del ciudadano medio para adoptar las decisiones más convenientes para la nación. Autores como Walter Lippmann y otros del "realismo democrático" (Westbrook, 1991) creían "intolerable e inviable la ficción de que cada uno de nosotros deberíamos adquirir una opinión competente sobre todos los asuntos públicos" (Lippmann, [1922] 2003: 43); no existe un "ciudadano omnicompetente" capaz de decidir lo más adecuado de modo racional, técnico e informado, porque "el mundo que debemos afrontar políticamente queda fuera de nuestro alcance, visión y comprensión” (Lippmann, [1922] 2003: 41). 
Así las cosas, el individuo ha solucionado esta incapacidad creando "pseudoentornos" (pesudo-environments) cognitivos, es decir, "representaciones del entorno" que le permiten formarse una imagen cabal, pero "ficticia" de aquellos aspectos del mundo sobre los que no tiene experiencia directa. En el capítulo "The World Outside and the Pictures in our Heads", explica Lippmann que la mayor parte del conocimiento sobre el mundo exterior no procede de la experiencia directa del individuo, sino del relato que otras personas le han hecho de ese mundo exterior.

De aquí derivaría el riesgo advertido por estos autores de que el sistema democrático descansa sobre las decisiones inexpertas del hombre medio, sometido a preguntas para las que no tiene respuestas y ante las que solo puede decidir arbitrariamente, de acuerdo con sus emociones y sentimientos. Esta es la razón por la que autores como Lippmann enfatizaron la función social de la prensa como modeladora de la opinión pública, hasta el punto de llegar a afirmar que, en sentido estricto, "la crisis actual de la democracia es una crisis de su periodismo" (Lippmann, [1920] 2011: 7).

El actual problema de la desinformación y las llamadas fake news representa una vieja amenaza para la formación de la opinión pública. Ya Walter Lippmann definía la propaganda como una actividad consistente en que "un grupo de hombres, capaces de evitar que la gente conozca los hechos de manera directa, manipulan las noticias relacionadas con ellos para adaptarlas a sus propósitos" (Lippmann, [1922] 2003: 51).

El hecho es que el Eurobarómetro de abril de 2018 recoge el inquietante dato de que el $37 \%$ de los europeos asegura recibir al menos una noticia falsa al día, y el $31 \%$, una vez a la semana; simultáneamente, el $83 \%$ de los europeos consideran las fake news una amenaza para la democracia. Estos mensajes simbólicos se caracterizan por un acusado contenido emocional y son lanzados a la esfera pública en contextos sociales muy polarizados bajo la apariencia de informaciones periodísticas.

Aunque las razones que explican los acontecimientos políticos acecidos en 2016 son muy complejas y no se pueden reducir a una sola causa (la crisis financiera desencadenada en 2008, las injusticias sociales derivadas de las dificultades de supervivencia para las clases sociales más desfavorecidas, la desafección ciudadana hacia la elite política y económica, la globalización y, evidentemente, el influjo de las redes sociales en la sociedad digital), la influencia de las campañas de desinformación puede haber desempeñado un papel determinante en las oscilaciones de la opinión pública.

Recientemente, Facebook ha admitido que 126 millones de americanos recibieron noticias falsas durante dicha campaña electoral (The Washington Post, 2017). Hemos sabido, igualmente, que los "trolls rusos" publicaron más de 80.000 entradas en Facebook entre 2015 y 2017. Según las informaciones disponibles, en un principio esas entradas fueron vistas por 29 millones de americanos, pero Facebook calcula que su propagación alcanzó al 40\% del total de la población. Los medios ya han comenzado a hablar de "víctimas" (La Nación, 2017).

A veces los mensajes simbólicos proceden de emisores conocidos con la capacidad de introducir en el debate público mensajes basados en datos falsos o en juicios de valor. La afición del presidente Trump a difundir mensajes de este tipo a través de la red social Twitter es un buen ejemplo. Durante la campaña electoral fueron propagados sus mensajes referentes a la inmigración con afirmaciones como: "Nuestro país ha perdido completamente el control de la inmigración ilegal y criminal”; "Nuestro país es un escenario criminal dividido, y solo puede ir a peor”; "Debemos parar la máquina criminal y asesina que es la inmigración ilegal”; "Clinton quiere inundar nuestro país con inmigrantes sirios de los que no sabemos casi nada. El daño es descomunal (massive)”. 
Esta realidad, que actualmente acapara gran parte de los análisis científicos, encauza el debate hacia la libertad individual y la amenaza que supone para las bases del sistema democrático. Según el director del Consejo Europeo de Relaciones Exteriores, Mark Leonard, los algoritmos secretos de las grandes empresas tecnológicas están determinando cómo percibimos el mundo, y está produciendo una creciente dificultad para que las personas tomen decisiones conscientes (Leonard, 2017).

El grado de influencia de la falsa noticia del apoyo del Papa Francisco a Donald Trump en la toma de decisión del voto es imposible de medir, a pesar de que algunos análisis apuntan en esa dirección (Bharat, 2017). Lo que parece fuera de toda duda es de que se trata de un mensaje diseñado deliberadamente para interpelar emocionalmente al electorado y condicionarlo en su libertad de voto, dado que tendemos a considerar "verdadera la visión que concuerde con nuestros sentimientos. Cuando creemos que algo tiene que ser verdad, casi siempre encontramos casos en los que lo es, o personas que lo creen así" (Lippmann, [1922] 2003: 131-136).

La posverdad, por lo tanto, nos introduce en el terreno de la percepción subjetiva de la realidad, donde las creencias y las convicciones individuales pesan más que la objetivad de los hechos. Aunque los datos pongan en evidencia el error de una creencia, el ser humano tenderá no a dudar de ella, sino de los hechos que la cuestionan. Este fenómeno fue descrito por Festinger, Riecken y Schacter en la obra When Prophecy Fails, donde leemos: "A man with a conviction is a hard man to change. Tell him you disagree and he turns away. Show him facts or figures and he questions your sources. Appeal to logic and he fails to see your point" (Festinger, Riecken, \& Schacter, 1956).

Poco después, Leon Festinger formularía la "Teoría de Disonancia Cognitiva" (A cognitive disonance, 1957), donde explica el mecanismo psicológico que se desencadena en el ser humano que, enfrentado a un conflicto -disonancia- entre sus creencias y los hechos, tratará de interpretarlos de modo congruente con su opinión. Este mecanismo de defensa podría llevarle a idear explicaciones o razonamientos que eliminen la incoherencia y refuercen sus convicciones en detrimento de los datos objetivos.

\section{Breve apunte sobre el Brexit}

En nuestra opinión, el resultado del Brexit no se puede atribuir exclusivamente a la influencia de las tecnologías Smart Data empleadas por Cambridge Analytica, sino al éxito que estas han tenido por ser utilizadas en un clima de euroescepticismo largamente difundido entre la sociedad británica. Por lo tanto, el contexto social de posverdad no se circunscribe a las inmediaciones temporales del referéndum, sino a un terreno abonado desde hace tiempo para que germinaran estos mensajes.

El profesor Chistoph Meyer, del King's College de Londres, afirma en este sentido que, en el caso del referéndum del Brexit, el resultado no se puede atribuir únicamente a la campaña política, sino "a los efectos negativos de la cobertura periodística de la Unión Europea sobre las creencias colectivas durante décadas. Mientras otros países también conocen el euroescepticismo, en Gran Bretaña es exclusiva la naturaleza del tratamiento periodístico de la integración europea" (Meyer, 2016). Según este analista, las acusaciones proferidas por los partidarios del Brexit durante la campaña calificando a "Bru- 
selas" de corruptos, opresivos, antidemocráticos, extranjeros, etc., tuvieron éxito entre una parte de la audiencia porque fueron difundidas durante años, especialmente por la prensa escrita.

Coincide con esta observación Andrew Crines, de la Universidad de Liverpool, quien asegura que "la campaña del referéndum comenzó hace mucho tiempo, aproximadamente 26 años” (Crines, 2016). Crines subraya el argumento de que los mensajes de los brexiters cayeron en un terreno abonado por el Partido Conservador, cuyos líderes han propagado durante décadas el mensaje de que "Europa nos maltrata". De modo correlativo, Meyer afirma que los partidarios de la permanencia en el bloque comunitario se lanzaron a la campaña sin tener en cuenta el "enorme déficit de conocimiento sobre la naturaleza de la Unión Europea” imperante en la sociedad británica (Meyer, 2016).

No deja de ser sintomático el hecho de que las preguntas más buscadas en Google en Reino Unido nada más conocerse el resultado del referéndum fueran, por este orden: “¿Qué significa abandonar la Unión Europea?”, “iQué es la Unión Europea?”, “¿Qué países forman la Unión Europea?”, “iQué pasa ahora que hemos abandonado la Unión Europea?” y “Cuántos países forman la Unión Europea?" (Google Trends, 24-06-2016, 13.24 h.).

De acuerdo con los sondeos de opinión llevado a cabo por IPSOS MORI en las fechas inmediatas al referéndum, la percepción mayoritaria de la opinión pública británica hacia problemas tan complejos como la inmigración o la economía fue coincidente con los asuntos enfatizados por los brexiters durante la campaña.

Preguntados los británicos sobre qué porcentaje del presupuesto comunitario europeo estaba destinado a sufragar a los funcionarios de las instituciones europeas, los encuestados contestaron de media que el $27 \%$ del presupuesto (es decir, algo más de 38 mil millones de euros), cuando en realidad se destina el 6\% (8 mil millones y medio de euros). Es importante esta diferencia porque uno de los eslóganes más repetidos durante la campaña estuvo relacionado con el oneroso mantenimiento de la burocracia comunitaria.

También resultó deformada la imagen de la inversión procedente de la Unión Europea en el Reino Unido, cifrada por los encuestados en el $30 \%$, cuando en realidad es del $48 \%$, 18 puntos por encima de la creencia popular; sin embargo, estos mismos encuestados pensaban que la inversión procedente de China en el Reino Unido era del 19\%, cuando en realidad es del 1\% (IPSOS MORI, 2016).

En resumen, la fotografía tomada por IPSOS MORI nos muestra, en la fecha del referéndum, una opinión pública británica abonada para que germinaran las semillas ideológicas de los partidarios del Brexit. La percepción de la realidad que muestran estos estudios inclina claramente la balanza hacia el lado de los partidarios de la desconexión.

\section{Conclusión}

Una lectura contemporánea del "Mito de la Caverna" nos ayudará a entender la posverdad como un problema de percepción de la realidad. Recordemos sucintamente que el filósofo ateniense situó a unos prisioneros en el interior de una caverna. Allí, retenidos en la misma posición desde su nacimiento, observan frente a ellos sombras de objetos que la luz de una antorcha colocada a sus espaldas proyecta sobre la pared. No pueden girar sobre sí mismos, de modo que no alcanzan a ver dichos objetos. La única referencia que tienen de ellos es el reflejo impreciso de sus sombras. Sin embargo, como no 
han visto otra cosa, los prisioneros confunden las sombras con la realidad misma. Actualizando el mito platónico, diríamos que los prisioneros de la caverna de Platón viven en la posverdad.

La posverdad consiste en un contexto social propicio para que los hechos objetivos (logos) sean relegados a un segundo plano del debate público por la irrupción de mensajes simbólicos con una acusada carga emocional (pathos). De donde se infiere que el éxito de estos mensajes depende de cómo sean interpretados por los ciudadanos en lo que Thomson (1997) llama "proceso de apropiación hermenéutica" de la realidad. Además, como señala José Ortega y Gasset (1914), el hombre está en un sistema de creencias que configura su estructura mental y que se impone en el proceso de atribución de significados a las cosas que percibe (realidad patente y realidad latente).

Somos seres sociales que no partimos de cero cuando nos enfrentamos al mundo; al contrario, desde la infancia hemos recibido paulatinamente, en un prolongado proceso de culturización, una estructura de interpretación del mundo.

\section{Referencias bibliográficas}

Bernays, E. (2010 -1928-). Propaganda. Barcelona: Melusina.

Bharat, K. (27-04-2017). How to Detect Fake News in Real-Time. NewCo. Disponible en: https://shift.newco.co/ how-to-detect-fake-news-in-real-time-9fdae0197bfd

Crines, A. (06-2016): “The rethoric of the EU referendum campaign". Referendum Analysis: http://www.referendumanalysis.eu/eu-referendum-analysis-2016/section-5-campaign-and-political-communication/the-rhetoric-of-the-eu-referendum-campaign/ (30-11-2017).

Davies, W. (24-08-2016): “The Age of Post-Truth Politics”. The New York Times: https://www.nytimes.com/2016/08/24/opinion/campaign-stops/the-age-of-post-truth-politics.html?_r=0 (15-05-2017).

European Commision (2018). Fake News and Disinformation online. Flash Eurobarometer, 464, abril. Disponible en: http:// ec.europe.es/commfrontoffice/publicopinion

Festinger, L., Riecken, H. \& Schacter, S. (1956): When Prophecy Fails. Minneapolis, Estados Unidos: University of Minnesota. Financial Times (2016). Brexit poll tracker. Finnancial Times, 23-06-2016. Disponible en: https://ig.ft.com/sites/ brexit-polling/

Gessen, M. (13-12-2016): “The Putin Paradigm”. The New York ReviewofBooks: http://www.nybooks.com/daily/2016/12/13/ putin-paradigm-how-trump-will-rule/ (15-05-2017).

Glencross,A.(2016): WhytheUKVotedforBrexit.London:PalgraveMacmillan.doi:https://doi.org/10.1057/978-1-137-59001-5

IPSOS MORI (2016). EU Referendum. IPSOS MORI: Disponible en: https://es.slideshare.net/IpsosMORI/ european-union-the-perils-of-perception

IPSOS MORI (2016). Perceptions are not reality: what the world gets wrong. IPSOS MORI. Disponible en: https://www.ipsos. com/ipsos-mori/en-uk/perceptions-are-not-reality-what-world-gets-wrong 
La Nación (31-10-2017): “Las noticias falsas rusas en Facebook llegaron a 126 millones de estadounidenses”. La Nación: http://www.lanacion.com.ar/2077863-las-noticias-falsas-rusas-en-facebook-llegaron-a-126-millones-de-estadounidenses (30-11-2017).

Lasswell, H. (1935). "The Person: Subject and Object of Propaganda”. The Annals of the American Academy of Political and Social Science, v. 179(1), 187-193.

Leonard, M. (2017): “The ilusion of Freedom in the Digital Age”. Project Syndicate: https://www.project-syndicate.org/commentary/technology-big-data-dystopia-by-mark-leonard-2017-11 (03-11-2017).

Lippmann, W. (2011 -1920-). Libertad y prensa. Madrid: Tecnos.

Lippmann, W. (2003 -1922-). La opinión pública. Madrid: Langre.

Marcus, R. (01-12-2016): Welcome to the post-truth presidency. The Washington Post. https://www.washingtonpost.com/ opinions/welcome-to-the-post-truth-presidency/2016/12/02/baaf630a-b8cd-11e6-b994-f45a208f7a73_story.html?utm_ term=.b3f1331e4d3d (15-05- 2017).

Ortega y Gasset, J. (2004 -1914-). Meditaciones del Quijote. En Obras completas (vol. 1, pp. 747-823). Madrid: Taurus / Fundacióin José Ortega y Gasset.

Oxford Dictionary. (2016, noviembre 16). Post-Truth. https://www.oxforddictionaries.com/press/news/2016/12/11/ WOTY-16 (09-05-2017).

Real Clear Politics (2016). General Election: Trump vs. Clinton, 08-11-2016. Disponible en: http://www.realclearpolitics. com/epolls/2016/president/us/general_election_trump_vs_clinton-5491.html\#polls

Remnick, D. (-02-09-2016): “Introducing a New Series: Trump and the Truth”. The New Yorker: http://www.newyorker.com/ news/news-desk/introducing-a-new-series-trump-and-the-truth (09-05-2017).

The Economist (10-09-2016): "Art of the lie". http://www.economist.com/news/leaders/21706525-politicians-have-always-lied-does-it-matter-if-they-leave-truth-behind-entirely-art?fsrc=scn/tw/te/pe/ed/artofthelie (15-05-2017).

Thomson, J. B. (1998 -1997-). Los medios y la modernidad. Una teoría de los medios de comunicación. Barcelona: Paidós.

Westbrook, R. B. (1991). John Dewey and American Democracy. Ithaca: Nueva York. 